\title{
Radio emission from ultra-high energy cosmic-ray showers after reflecting on the Earth.
}

\author{
Daniel García-Fernández*, Jaime Alvarez-Muñiz, Washington Rodrigues Carvalho \\ Jr. \\ Departamento de Física de Partículas \& Instituto Galego de Física de Altas Enerxías \\ Universidade de Santiago de Compostela, 15782, Santiago de Compostela, Spain \\ E-mail: sirzidegmail.com, jaime.alvarezmuniz@gmail.com, \\ carvajregmail.com
}

\section{Harm Schoorlemmer}

University of Hawaii at Manoa, Department of Physics and Astronomy

Honolulu, Hawaii 96822, USA

E-mail: harmscho@phys.hawaii.edu

\section{Enrique Zas}

Departamento de Física de Partículas \& Instituto Galego de Física de Altas Enerxías Universidade de Santiago de Compostela, 15782, Santiago de Compostela, Spain

E-mail: zasefpaxp1.usc.es

\begin{abstract}
We present the calculation of coherent radio pulses emitted by extensive air showers induced by ultra-high energy cosmic rays accounting for reflection on the Earth's surface. Our work is motivated by the detection of pulsed events in the ANITA experiment compatible with cosmicray origin after reflection on the ice cap at the South Pole. The properties of the radiation are discussed in detail emphasising the effects of reflection on the intensity, frequency and angular distribution of the signal. Our results are obtained with a new version of the ZHAireS simulation code, accounting for reflection on a surface. These calculations are of relevance in studies of existing and future radio detection experiments looking at the Earth from mountain tops, balloons and satellites.
\end{abstract}

The 34th International Cosmic Ray Conference,

30 July- 6 August, 2015

The Hague, The Netherlands

\footnotetext{
* Speaker.
} 


\section{Introduction}

The ANITA experiment discovered 14 radio pulses reflected on the polar ice cap from air showers extending up to the $\mathrm{GHz}$ range with its first balloon borne antenna system [1]. ANITA, operating within a frequency band between 200 and $1200 \mathrm{MHz}$, had been conceived for detecting radio emission from neutrino interactions.

After this discovery, and following the same idea, observatories on top of mountains have been planned. One of them is the TAROGE [2] experiment, on the mountains of Taiwan's east coast. So far, only a prototype (TAROGE-1) has been built, consisting of 12 log-periodic dipole array antennas for 110-300 MHz, at an altitude of $\sim 1 \mathrm{~km}$. Besides the altitude of the detector antennas, the capital difference with the ANITA experiment is the presence of seawater as the reflecting surface.

Reflection changes the arrival times and therefore the coherence of the radio pulse from the shower and implies a reduction of the signal due to the Fresnel coefficients. These effects have to be taken into account to understand experiments that observe reflected radiation induced by showers from mountain tops, balloon payloads [3] such as the next ANITA flight and EVA [4] or from satellite payloads as SWORD [5].

In this work we use a modified version of the ZHAireS code [6] to calculate the emission from air showers after reflection on a flat surface. A detailed discussion can be found in [7].

\section{Geometry for reflected events}

Observing reflected pulses a large atmospheric volume can be monitored with a single detector. Therefore, the most interesting geometry is given by air showers that impact Earth's surface at large zenith angles. The basic geometry of the problem is sketched in Fig. 1. The reflective surface will be approximated by a plane. $\theta$, the shower angle, is defined with respect to the $z$-axis. We define the off-axis angle $\psi$ in the right panel of Fig. 1 to describe the angular deviation of the emitted radiation with respect to the shower axis ${ }^{1}$.

\section{Introducing reflection of radio waves in ZHAireS}

ZHAireS [6] is a simulation program that has been developed combining the AIRES package for air shower simulations [8] with the "ZHS algorithm" [6, 9]. The contribution from each particle track to the radio pulse at any given position and time is calculated and added to the total electric field. To calculate the travel time of the radiated pulse we perform a numerical integration to account for the variation of the index of refraction and assume the emitted radiation travels in a straight line. As shown in [7] this is a good approximation up to $\theta \sim 85^{\circ}$.

We have modified the ZHAireS code to deal with the reflection on a flat surface. For each rectilinear track element we first find the point on the reflection surface and the angle of emission with respect to the track so that the emitted ray propagates first to the reflection point and then upwards towards the observer at a fixed position. The time delay is easily calculated integrating the travel time over the total path of the ray before and after the reflection.

\footnotetext{
${ }^{1} \psi$ as depicted in Fig. 1 (right) is also used to refer to the location of the observer.
} 

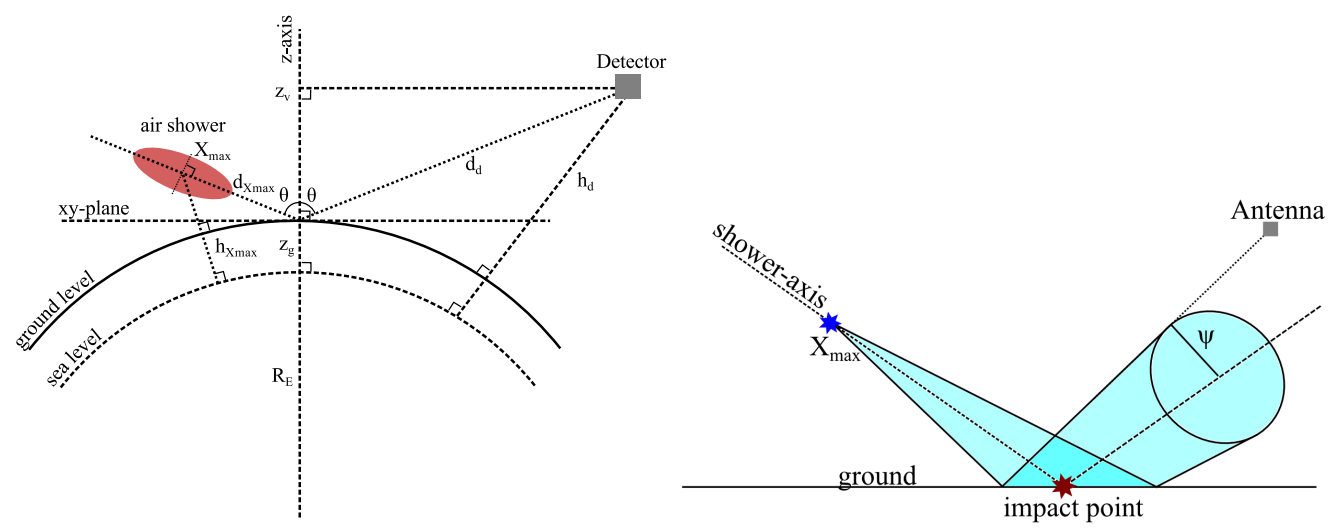

Figure 1: Left: Geometry for reflected signals from air showers (see text for details). Right: To describe a location of an antenna we use the off-axis angle $\psi$ between the shower axis and the line joining $X_{\max }$ and the antenna position.

At each reflection point the Fresnel coefficients are applied to the time-domain electric field to calculate the attenuation of the components with polarization parallel, $r_{\|}$, and perpendicular, $r_{\perp}$, to the reflection plane. Introducing the effective relative permittivity as

$$
\tilde{\varepsilon}_{r}=\frac{\varepsilon}{\varepsilon_{0}}+i \frac{\sigma}{\varepsilon_{0} \omega}
$$

where $\varepsilon$ is the complex permittivity of the medium, $\sigma$ its conductivity and $\omega$ the angular frequency, the boundary conditions for plane waves remain identical $[10,11]$ and the functional form of the Fresnel coefficients is similar to that of the usual lossless case. We consider the medium labeled as 1 as air, with a real permittivity and without conductivity, while in general medium 2 (ice or sea water) has a complex relative permittivity $\tilde{\varepsilon}_{r}$. Therefore,

$$
r_{\perp}=\frac{n_{1} \cos \theta_{i}-\sqrt{\tilde{\varepsilon}_{r, 2}} \cos \theta_{t}}{n_{1} \cos \theta_{i}+\sqrt{\tilde{\varepsilon}_{r, 2}} \cos \theta_{t}}, \quad r_{\|}=\frac{n_{1} \cos \theta_{t}-\sqrt{\tilde{\varepsilon}_{r, 2}} \cos \theta_{i}}{n_{1} \cos \theta_{t}+\sqrt{\tilde{\varepsilon}_{r, 2}} \cos \theta_{i}},
$$

where the incident angle $\theta_{i}$, and the transmitted angle $\theta_{t}$ are related by $n_{1} \sin \theta_{i}=\operatorname{Re}\left[\sqrt{\tilde{\varepsilon}_{\mathrm{r}, 2}}\right] \sin \theta_{\mathrm{t}} \equiv$ $\mathrm{n}_{2} \sin \theta_{\mathrm{t}}$. The last equivalence can be used to make Eq. 3.2 look identical to the usual lossless case.

\section{Simulations for a high altitude detector}

We adopt a set of observers that resembles a high altitude balloon experiment over Antarctica, such as the ANITA flights or the planned EVA mission [4]. We place antennas at a fixed altitude $h_{d}=36 \mathrm{~km}$ above sea level, and choose the reflecting surface to be at $z_{g}=2 \mathrm{~km}$ above sea level (see Fig. 1). We adopt a refractive index of $n_{2}=1.31$, and a real effective permittivity [12], consistent with ANITA measurements of the reflected image of the Sun [13] on the Antarctic firn. The geomagnetic field is chosen to have a typical value of $55 \mu \mathrm{T}$ and an inclination of $-70^{\circ}$. We generated proton showers with zenith angles $\theta=\left\{57^{\circ}, 64^{\circ}, 71^{\circ}, 78^{\circ}, 85^{\circ}\right\}$ and azimuth such that they always arrive from the geomagnetic west. For each zenith angle, we generate air showers with energies 

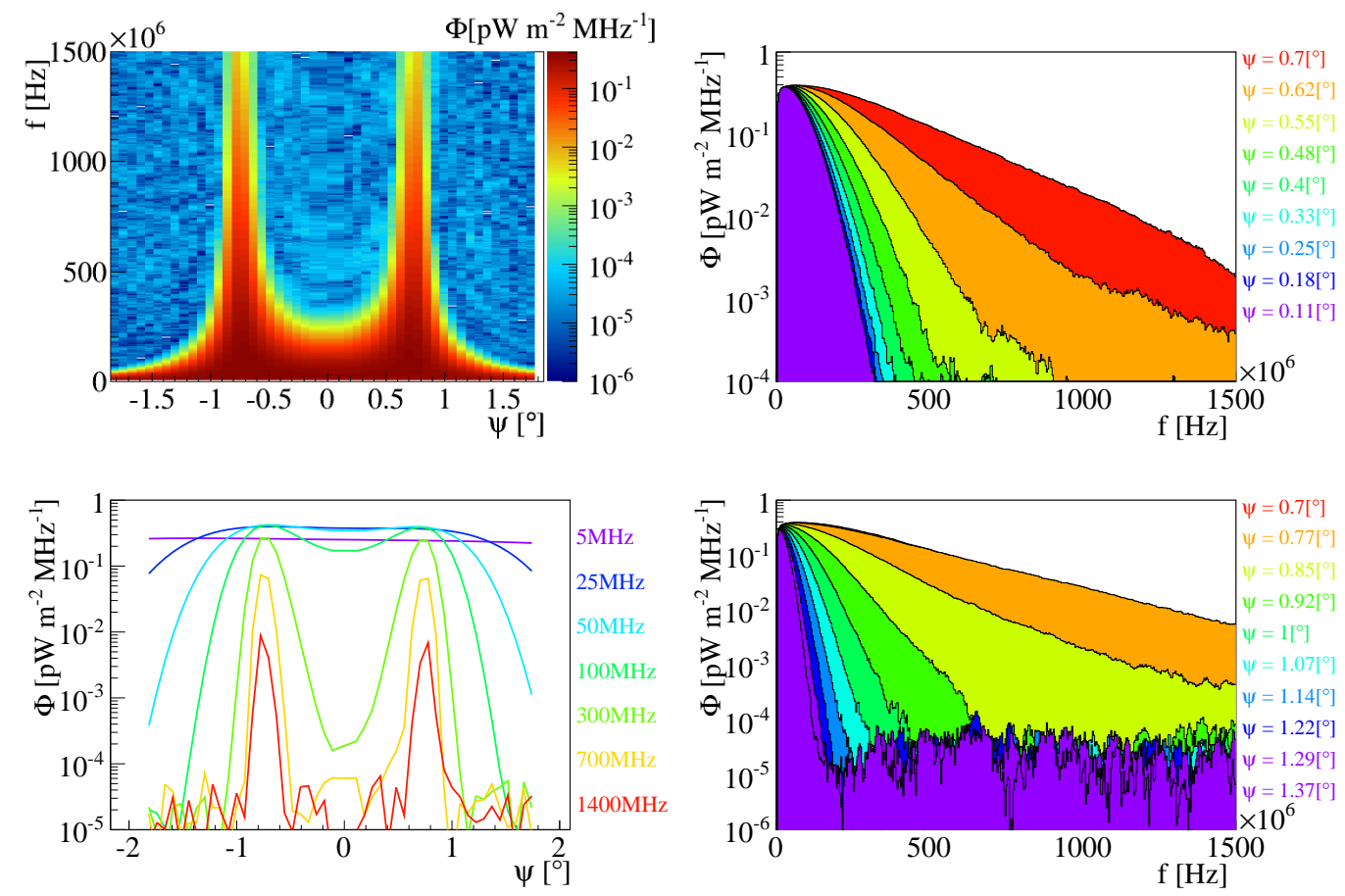

Figure 2: Top left panel: distribution of the radio signal (flux density $\Phi$ ) as function of the off-axis angle $\psi$ and frequency $f$ for an air shower with $\theta=71^{\circ}$ and $\log _{10}(E / \mathrm{eV})=18.4$. In the bottom left panel we show the distribution of the radio signal as a function of the off-axis angle at various frequencies. In the right panels we show the radio signal distribution as a function of frequency, in the top right panel for off-axis angles equal or smaller than the Cherenkov angle at the $X_{\max }$ of the shower, i.e. $\psi \leq 0.7^{\circ}$, while in the bottom right panel for $\psi \geq 0.7^{\circ}$.

$E=\left\{10^{17.8}, 10^{18.4}, 10^{19}, 10^{19.6}\right\} \mathrm{eV}$. We select simulations that have a shower maximum similar to the average $\left\langle X_{\max }\right\rangle_{\text {Auger }}$ observed at the Pierre Auger Observatory. To do so, we pre-simulate seven air showers per configuration with different random seeds and we select the air shower closest to $\left\langle X_{\max }\right\rangle_{\text {Auger }}$. The shower simulation is run with AIRES using QGSJETTII.03 hadronic model interactions with a thinning level of $10^{-5}$ [8].

To illustrate some of the typical features of the radio signal, we display in Fig. 2 the flux density $\Phi$ as a function of frequency and off-axis angle $\psi$ for an air shower with zenith angle $\theta=71^{\circ}$ and an energy $E=10^{17.8} \mathrm{eV}$. The flux density in units of $\mathrm{pW} \mathrm{m}{ }^{-2} \mathrm{MHz}^{-1}$ is defined as the power spectrum at a fixed frequency $f$ averaged over a period of $10 \mathrm{~ns}$. In the top left panel of Fig. 2 we show the two-dimensional distribution as a function of $\psi$ and $f$ which displays coherent properties and is clearly beamed around the Cherenkov angle at $\psi \sim 0.77^{\circ}$. This can be better appreciated in the bottom left panel where we show the off-axis angle distributions for different frequency components of the pulse. As the frequency increases the radiation adds coherently only within a smaller angle off the Cherenkov cone. In the right panels in Fig. 2 we show the spectral shape of the flux density for a variety of observation off-axis angles. At very low frequencies $(f<10 \mathrm{MHz}$ ) the flux density increases until it reaches a maximum in the range $f \sim 10-150 \mathrm{MHz}$ and then decreases with an exponential fall-off to first order. A very important feature is illustrated 

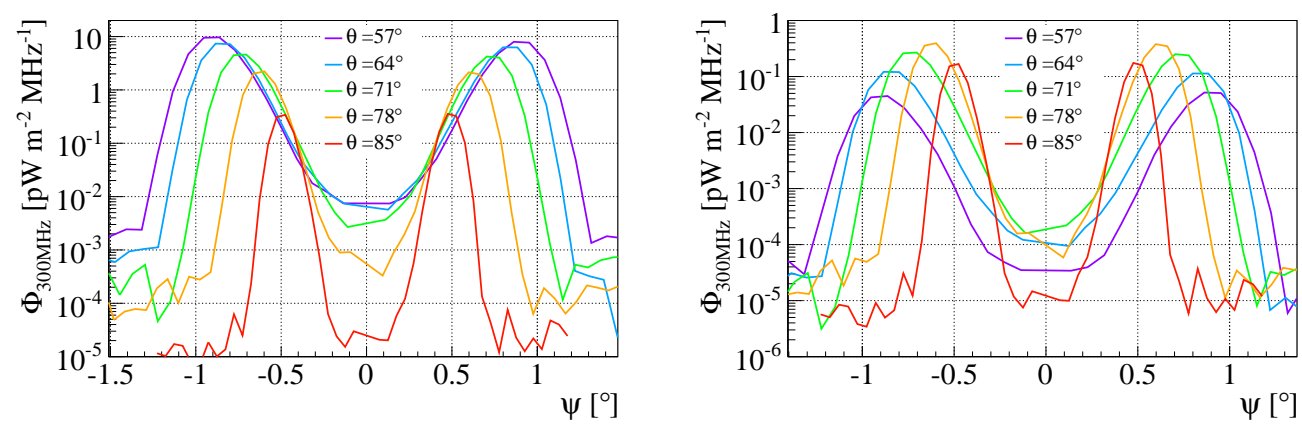

Figure 3: Comparison of the flux-density $\Phi$ at $f=300 \mathrm{MHz}$ as a function of the off-axis angle $\psi$ before (left) and after applying Fresnel reflection coefficients (right). Different sets of curves correspond to different zenith angles $\theta$ as labeled. The simulations have an energy of $10^{18.4} \mathrm{eV}$.

in the right panels, the steepness of the fall-off has a clear dependence on the off-axis angle $\psi$ of the detector. This dependence is key to the energy determination of UHECRs with ANITA as pointed out in [14] and shown in [22].

It is interesting to explore how the radiation changes with zenith angle for a primary particle with fixed energy. As the zenith angle increases the flux density $\Phi$ decreases. This can be seen in the left panel of Fig. 3, displaying $\psi$ at $f=300 \mathrm{MHz}$ as a function of $\psi$ for an air shower induced by a primary particle with energy $E=10^{18.4} \mathrm{eV}$. The dominant effect in the decrease is the increasing overall distance to the detector with $\theta$. Other effects however compensate this decrease. The angle $\alpha$ of the shower axis to the Earth's magnetic field at the South Pole increases in the range of $\theta$ shown in Fig. 3, and the geomagnetic contribution is known to scale with $\sin \alpha$. Also, showers of increasing $\theta$ develop in a less dense atmosphere where the geomagnetic contribution to the electric field is expected to be increasingly larger [15, 6]. The net result, including other more subtle effects [16] is a decrease of $\Phi$ with $\theta$.

To illustrate the importance of accounting for the Fresnel coefficients they were artificially set to 1 in the simulations shown in the left panel of Fig 3 (with the Fresnel coefficients ignored), while in the right panel they are taken into account using Eqs. 3.1 and 3.2 with real $\varepsilon$ and with $\sigma=0$. Comparing both panels, the peak value of the flux density is largest at relatively high zenith angles $\left(\theta \sim 80^{\circ}\right)$ when the Fresnel coefficients are accounted for, contrary to what is seen in the left panel where the peak value of $\Phi$ is achieved at the smallest zenith angles. This suggests that detection can be expected to be most favourable for $\theta$ around $80^{\circ}$. A thorough calculation of the acceptance integrating over area and solid angle [3] should also account for the reduction of the Cherenkov angle as the zenith angle rises and for the directionality of the detection system [22] (see left panel of Fig. 3).

From the set of simulations we have examined the energy dependence of the radio signal. As before, we use the flux density at a reference frequency $f=300 \mathrm{MHz}$ for a shower of $\theta=71^{\circ}$. In Fig. 4 we select three off-axis angles and plot the flux density as a function of the primary particle energy. We fit a simple linear function to the dependence of $\log _{10} \Phi$ on $\log _{10} E$ and find a slope that is consistent with 2 . This confirms that the received flux density scales quadratically with the 


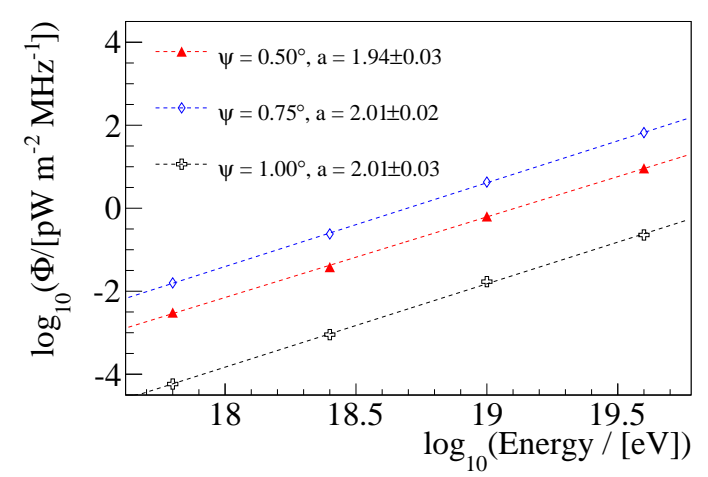

Figure 4: The flux density $\Phi$ at $f=300 \mathrm{MHz}$ as a function of the energy of the primary particle for three off-axis angles. The results of fitting a straight $\operatorname{line} \log _{10} \Phi=a \log _{10}(E / \mathrm{eV})+\mathrm{b}$ are shown.

primary energy and the amplitude of the electric field scales linearly with it. This is not surprising since for a coherent signal it is expected that the amplitude of the electric field scales with the number of electrons in the shower which is proportional to the energy of the primary particle. We have verified that the quadratic relation between flux density and energy of primary cosmic ray particle holds for all the zenith angles and all considered frequencies in our simulation set. This has important consequences since measuring the flux density at a given frequency and off-axis angle provides a measurement of the energy of the air shower. In practice the off-axis angle can be related to the exponential fall-off of the flux density as can be seen in Fig. 2. This relation is key to the energy destermination of UHECRs with ANITA [14]. This means that it is in principle possible to deduce the primary energy from a single location as long as the exponential fall-off of the spectrum can be determined. This procedure is applied in [22] to estimate the energy of the 14 CR events detected by ANITA with which a first determination of the CR spectrum based on radio observations only was done.

\section{Simulations for observatories on mountain tops}

As in the case of ANITA, we have also performed simulations with the ZHAireS version for reflected events on seawater for the planned TAROGE experiment. In a first step, we have calculated the reflected electric field without Fresnel coefficients. After that, we apply the Fresnel coefficients (Eq. 3.2) for the field of the shower as a whole using as incident angle the sum of the shower zenithal angle and the observer's off-axis angle, $\theta_{i}=\theta+\psi$. Although it is more precise to apply the coefficients for the field of each individual track as in the previous section, the difference between the two procedures of accounting for the Fresnel coefficients is negligible [17].

We have simulated two $10^{19} \mathrm{eV}$ showers of $60^{\circ}$ and $80^{\circ}$ of zenithal angle, both of them coming from the north. Observers lie on the north-south line at a constant $z$ coordinate (not altitude, see Fig. 1) of $1 \mathrm{~km}$, each defining a single off-axis angle. The magnetic field has been chosen using a WMM (World Magnetic Model) with the approximate coordinates of TAROGE $\left(23.5^{\circ} \mathrm{N}, 121^{\circ} \mathrm{E}\right.$, $1 \mathrm{~km}$ above sea level), which results in a magnetic field with magnitude $44.6 \mu \mathrm{T}$ declination $-3.8^{\circ}$ and inclination $34.4^{\circ}$. 

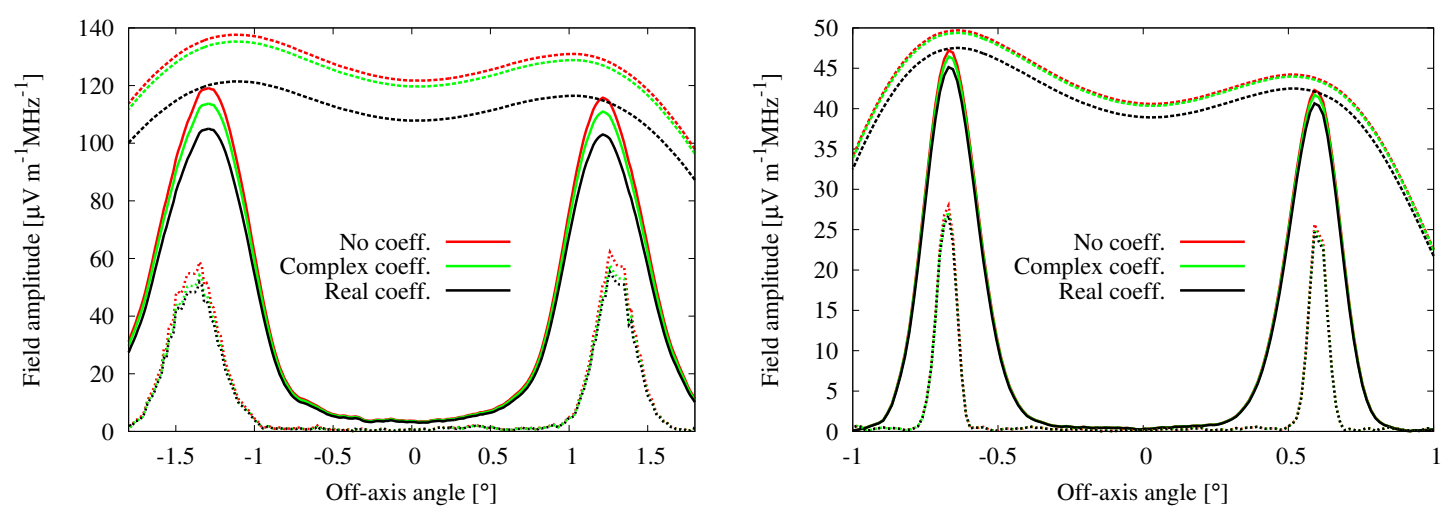

Figure 5: Amplitude of the E-W component of the electric field for a $10^{19} \mathrm{eV}$ shower coming from the magnetic North. Observation frequencies are, from top to bottom, 50, 300 and $900 \mathrm{MHz}$. We plot the components without reflection coefficient (red), with coefficient for complex effective permittivity (blue) and with coefficient for real permittivity (black) as given by Eq. 3.1. Left: $60^{\circ}$ shower. Right: $80^{\circ}$ shower.

For the effective permittivity in Eq. 3.1 we will use the Stogryn model proposed in [18], which gives good results for the measurements between 200 and $1400 \mathrm{MHz}$ [19] and higher frequencies [20]. The model is dependent on the temperature and salinity of the water. Typical reference values are $20{ }^{\circ} \mathrm{C}$ for the temperature and $35 \mathrm{~g} / \mathrm{kg}$ for the salinity. Under these conditions and between 50 and $1000 \mathrm{MHz}$ seawater is well approximated by a medium with relative permittivity $\varepsilon_{r} \sim 69$ and conductivity $\sigma \sim 5 \Omega^{-1} \mathrm{~m}^{-1}$. Increasing (Decreasing) the salinity or temperature also increases (decreases) the conductivity and the reflection is therefore enhanced (reduced).

As with the high altitude simulations, we assume a flat surface for reflections. Corrections due to sphericity and roughness of the sea surface can be taken into account with the methods presented in [21].

In Fig. 5 we plot the results for the amplitude of the East-West (E-W) component in showers of $60^{\circ}$ (left) and $80^{\circ}$ (right) zenith angle showers. The E-W component dominates the emission due to the geomagnetic effect. Amplitudes are shown without Fresnel coefficient, with the coefficient for a lossless medium (real effective permittivity) and with the complex coefficient for a conductive and absorptive medium (complex effective permittivity as in Eq. 3.1). As seen in Fig. 5, a conductive medium reflects more than a pure dielectric, and therefore seawater reflects more signal than pure water or ice. Moreover, in this frequency range almost all the E-W component gets reflected. The lower the frequency and the higher the zenith angle, the closer the coefficient is to 1.

\section{Summary and conclusions}

We have implemented the treatment of surface reflections for radio signals from air showers, upgrading existing ZHAireS simulations. We have simulated radiation reflected on the Antarctic ice going towards a high altitude balloon and radiation reflected on the sea towards a mountain top observatory. We have shown the importance of accounting for the Fresnel coefficients. The special version of the ZHAireS code can be used to assess the design and feasability of future large exposure cosmic ray detectors based on the radio technique applied to air showers [3,5]. 


\section{Acknowledgements}

We thank Ministerio de Economía (FPA2012-39489), Consolider-Ingenio 2010 CPAN Programme (CSD2007-00042), Xunta de Galicia (GRC2013-024), Feder Funds and Marie CurieIRSES/ EPLA- NET (European Particle physics Latin American NETwork), 7th Framework Program (PIRSES- 2009-GA-246806). H.S. is supported by Office of Science, U.S. Department of Energy and N.A.S.A. We also thank CESGA (Centro de Supercomputación de Galicia) for computing resources.

\section{References}

[1] S. Hoover et al., Phys. Rev. Lett. 105, 151101 (2010).

[2] J. Nam, in proceedings of ICRC 2015, POS ( ICRC2015) 663 (2015).

[3] P. Motloch, N. Hollon, P. Privitera, Astrop. Phys. 54 (2014) 40-43.

[4] P.W. Gorham et al., Astrop. Phys. 35 (2011) 242-256.

[5] A. Romero-Wolf, et al., arXiv:1302.1263 [astro-ph].

[6] J. Alvarez-Muñiz, W.R. Carvalho Jr., E. Zas, Astrop. Phys. 35 (2012) 325-341.

[7] J. Alvarez-Muñiz, W.R. Carvalho Jr., D. García-Fernández, H. Schoorlemmer, E. Zas. Astrop. Phys. 66 (2015) 31-38.

[8] S.J. Sciutto, arXiv 99.11.331 (1999), http://www2.fisica.unlp.edu.ar/auger/aires

[9] D. García-Fernández, J. Alvarez-Muñiz, W. R. Carvalho Jr., A. Romero-Wolf, E. Zas, Phys. Rev. D 87, 023003 (2013).

[10] D. J. Griffiths, Introduction to Electrodynamics 3rd ed., Prentice Hall, New Jersey 1999.

[11] J. D. Jackson, Classical Electrodynamics, 3rd ed, Wiley, New York 1998.

[12] I. Kravchenko, D. Besson, J. Meyers, J. Glaciology 50 (2004) 171.

[13] D.Z. Besson et al., Radio Sci. 50 (2015), http://dx.doi.org/10.1002/2013RS005315.

[14] K. Belov and ANITA Collaboration, in proceedings of 5th International Workshop on Acoustic and Radio EEV Neutrino Detection Activities: ARENA 2012, AIP Conf. Proc. 1535 (2013) 209.

[15] O. Scholten, K. Werner, F. Rusydi, Astrop. Phys 29 (2008) 94-103

[16] J. Alvarez-Muñiz, W.R. Carvalho Jr., A. Romero-Wolf, M. Tueros, E. Zas, AIP Conf. Proc. 1535 (2013) 143

[17] J. Alvarez-Muñiz, W.R. Carvalho Jr., D. García-Fernández, H. Schoorlemmer, E. Zas (presented by E. Zas), in proceedings of ARENA 2014, 9-12 June 2014, Annapolis (USA)

[18] L.A. Klein, C.T. Swift, IEEE Transactions on Antennas and Propagation, Vol. AP-25, No.1, January 1977.

[19] D.H. Gadani, V.A.Rana, A.N. Prajapati, A.D Vyas, Indian Journal of Pure \& Applied Physics, Vol. 50, June 2012, pp. 405-410.

[20] W. Ellison et al., Radio Science, Volume 33, Number 3, Pages 639-648, May-June 1998.

[21] A. Romero-Wolf et al., Icarus 248 (2015) 463-477.

[22] H. Schoorlemmer et al., arXiv 1506.05396.

[23] H. Schoorlemmer et al., in proceedings of ICRC 2015, POS (ICRC2015) 272 (2015). 